Journal of the Operations Research

Society of Japan

Vol. 35, No. 2, June 1992

\title{
STATISTICAL CRITERIA FOR SEQUENTIAL INSPECTOR-LEADERSHIP GAMES
}

\author{
Rudolf Avenhaus \\ Akira Okada \\ University of the Federal Armed Forces Kyoto University
}

(Received January 23, 1991)

\begin{abstract}
This paper considers ways of modelling time dependent inspection problems where there is a possibility of false alarms. For this purpose, it is assumed that safeguards procedures are notified to the inspectee by the inspector and are prima facie plausible ones. This 'inspector-leadership principle' implies that under reasonable assumptions the inspectee's strategy is legal in the sense of complying with agreed rules. The main objective of our investigation is to derive simple criteria for the determination of optimal inspection procedures from the equilibrium conditions for non-cooperative non-zero sum two-person games. It can be shown that, given the appropriate assumptions, one can arrive at 'statistical' optimization criteria. In the simplest case one gets the global probabilities of the errors of the first and second kind and in more complex cases the average run lengths for legal and illegal inspectee behavior.
\end{abstract}

\section{Introduction}

With a few exceptions until now only time independent inspection problems were treated with the help of game theoretical models, or time dependent problems were transformed into time independent ones by use of appropriate assumptions. In addition, the few exceptions [4], [5], [7], [8] represented inspection problems where false alarms were not possible.

In this paper the question for suitable ways of modelling time dependent inspection problems is posed, and it is assumed that false alarms cannot be avoided. Furthermore, the 'inspector-leadership principle' is used, i.e., it is assumed that the inspector notifies his inspection procedure to the inspectee in a plausible way. In fact, this principle is used in various international safeguards systems; it guarantees under some additional assumptions at least in the time independent case that in equilibrium the inspectee behaves legally in the sense of complying with agreed rules.

The main objective of our investigation is to derive simple criteria for the determination of optimal inspection procedures from the equilibrium conditions for non-cooperative non-zero sum two-person games. For this purpose, we consider a class of inspection games where both players - inspector and inspectee - can act independently at a given number of discrete time steps. The set of pure strategies of the inspector is the set of statistical decision procedures based on observations of random variables at the various time steps. That of the inspectee consists of the choices between legal and illegal behavior, and an appropriate illegal strategy in the latter case. The game ends at any stage where the inspector decides that the inspectee behaved illegally, otherwise it continues until the last step where the inspector decides definitely whether or not the inspectee behaved legally.

If one assumes that time does not play a role in the players' evaluation of the possible outcomes of the game, then it suffices to consider the global probabilities of the errors of 
the first and second kind (false alarm and no detection of illegal action) as criteria for the determination of the equilibrium test procedure of the inspector. If one considers, however, an infinite sequence of discrete time steps and assumes that the payoffs to both players are discounted with time, then it can be shown that it suffices to use the average run lengths for legal and illegal inspectee behavior. This is a very satisfying result, since these criteria in fact are used for solving practical statistical problems.

The paper is organized as follows: Section 2 describes a general model of a sequential inspection game. Section 3 introduces the inspector-leadership principle and presents a general condition of a subgame perfect equilibrium point of the sequential inspectorleadership game. Section 4 investigates under what assumptions the sequential inspectorleadership game is equivalently reduced to the non-sequential inspector-leadership game. Section 5 analyzes the sequential inspector-leadership game in the infinite time horizon case. It is shown under some appropriate assumptions that we can use average run lengths for legal and illegal inspectee behavior as the criteria for the optimal test procedure of the inspector.

\section{A Sequential Inspection Game}

There are two players $I$ (inspector) and $O$ (inspectee). $M_{1}, \ldots, M_{n}$ are the (pure) action sets of the inspectee $O$ at stages $1, \ldots, n$, respectively. The action set of the inspector $I$ is the same at all stages and consists only of two elements, $A$ (alarm) and $\bar{A}$ (no alarm). We assume that the set of 'feasible' actions for the inspectee at every stage $t=2, \ldots, n$ may depend on his previous actions before stage $t$. In order to formulate such a general situation, we introduce a collection of mappings $\left\{\bar{M}_{t}\right\}_{t=1}^{n}$, where each $\bar{M}_{t}, t=1, \ldots, n$, maps its domain $D_{t-1} \subset M_{1} \times \ldots \times M_{t-1}$ into $2^{M_{t}}$, i.e., the class of all subsets of $M_{t}$. To every $\left(\mu_{1}, \ldots, \mu_{t-1}\right) \in D_{t-1}$, the map $\bar{M}_{t}$ assigns the set $\bar{M}_{t}\left(\mu_{1}, \ldots, \mu_{t-1}\right)$ of all feasible actions in $M_{t}$ for the inspectee at stage $t$ under the history $\left(\mu_{1}, \ldots, \mu_{t-1}\right)$. The domains $D_{0}, \ldots, D_{n-1}$ of the mappings $\bar{M}_{1}, \ldots, \bar{M}_{n}$ are defined inductively as follows:

(1) $D_{0}=\left\{\mu_{0}\right\}$ where $\mu_{0}$ means the empty history,

(2) for every $t=1, \ldots, n, D_{t}$ is the set of all $\left(\mu_{1}, \ldots, \mu_{t}\right)$ in $M_{1} \times \ldots \times M_{t}$ satisfying $\left(\mu_{1}, \ldots, \mu_{t-1}\right) \in D_{t-1}$ and $\mu_{t} \in \bar{M}_{t}\left(\mu_{1}, \ldots, \mu_{t-1}\right)$.

The domain $D_{t}$ of the mapping $\bar{M}_{t+1}$ can be interpreted as the set of all sequences of the inspectee's feasible actions from stage 1 to stage $t$.

For the purpose of illustration, we consider a material control problem, see, e.g., [1]. Here, $O$ tries to divert the amount $\mu_{t}$ of material at stages $t=1, \ldots, n$ such that $\Sigma_{t} \mu_{t}=\bar{\mu}$; the domain $D_{t-1}$ is defined by

$$
D_{t-1}=\left\{\left(\mu_{1}, \ldots, \mu_{t-1}\right) \mid \sum_{i=1}^{t-1} \mu_{i} \leq \bar{\mu}, \mu_{i} \geq 0, i=1, \ldots, t-1\right\}
$$

and the mapping $\bar{M}_{t}$ is defined by

$$
\bar{M}_{t}\left(\mu_{1}, \ldots, \mu_{t-1}\right)=\left\{\mu_{t} \mid \sum_{i=1}^{t-1} \mu_{i}+\mu_{t} \leq \bar{\mu}, \mu_{t} \geq 0\right\}
$$

for every $\left(\mu_{1}, \ldots, \mu_{t-1}\right) \in D_{t-1}$. The total amount $\bar{\mu}$ of material to be diverted is predeter- 
mined and known to both players.

$X_{1}, \ldots, X_{n}$ are the sets of signals (or observations) the inspector can receive at stages $1, \ldots, n$, respectively. For any $\left(\mu_{1}, \ldots, \mu_{t}\right) \in D_{t}$ and any $\left(x_{1}, \ldots, x_{t-1}\right) \in X_{1} \times \ldots \times X_{t-1}$ an observation $x_{t} \in X_{t}$ at stage $t$ is drawn by a chance move. The observation $x_{t}$ is a random variable with the conditional probability density $f_{t}\left(x_{t} \mid \mu_{1}, x_{1}, \ldots, x_{t-1}, \mu_{t}\right)$. In what follows, we assume that all $M_{t}$ and $X_{t}(t=1, \ldots, n)$ are finite dimensional Euclidean spaces or their subsets.

For every $t=1, \ldots, n, I_{t}(\cdot)$ and $0_{t}(\cdot)$ are real-valued functions on $D_{t}$. For every $\left(\mu_{1}, \ldots, \mu_{t}\right) \in D_{t}, I_{t}\left(\mu_{1}, \ldots, \mu_{t}\right)$ and $0_{t}\left(\mu_{1}, \ldots, \mu_{t}\right)$ represent the inspector's and the inspectee's payoffs, respectively, if the game stops at stage $t$. The stopping rule of the game will be explained below.

A sequential inspection game $\Gamma_{1}$, whose extensive form is sketched in Figure 1, is played as follows:

(1) At stage 1, the inspectee $O$ chooses an action $\mu_{1} \in M_{1}$ (not observable to the inspector $I$ ). An observation $x_{1}$ is drawn by a chance move according to the probability density function $f_{1}\left(\cdot \mid \mu_{1}\right)$. The observation $x_{1}$ becomes common knowledge to both players.

(2) The inspector chooses either $\bar{A}$ (no alarm), in which case the game continues to stage 2 , or $A($ alarm $)$, in which case the game terminates with payoffs $\left(I_{1}\left(\mu_{1}\right) ; O_{1}\left(\mu_{1}\right)\right)$.

(3) Inductively: for every $t=1, \ldots, n$, after the inspectee chose actions $\left(\mu_{1}, \ldots, \mu_{t-1}\right) \in$ $D_{t-1}$ and observations $\left(x_{1}, \ldots, x_{t-1}\right)$ were drawn, the inspectee chooses an action $\mu_{t} \in \bar{M}_{t}\left(\mu_{1}, \ldots, \mu_{t-1}\right)$ with complete knowledge of $\left(\mu_{1}, x_{1}, \ldots, \mu_{t-1}, x_{t-1}\right)$. Thereafter, an observation $x_{t}$ is drawn by a chance move according to the conditional probability density function $f_{t}\left(\cdot \mid \mu_{1}, x_{1}, \ldots, \mu_{t-1}, x_{t-1}, \mu_{t}\right)$. With observations $\left(x_{1}, \ldots, x_{t}\right)$ the inspector chooses either $\bar{A}$, in which case the game continues to stage $t+1$ if $t<n$ or terminates with payoffs $\left(\tilde{I}_{n}\left(\mu_{1}, \ldots, \mu_{n}\right) ; \tilde{O}_{n}\left(\mu_{1}, \ldots, \mu_{n}\right)\right)$ if $t=n$, or $A$, in which case the game terminates and the payoffs are $\left(I_{t}\left(\mu_{1}, \ldots, \mu_{t}\right) ; O_{t}\left(\mu_{1}, \ldots, \mu_{t}\right)\right)$.

If the inspectee's actions are given at all his moves, the inspector is faced with an 'optimal stopping problem' in the sequential inspection game $\Gamma_{1}$ (see [3]). The inspector has to decide when to raise an alarm, i.e., stop the game. However, unlike other wellstudied game versions of the stopping problem, the second player (the inspectee) has no means to stop the game.

\section{The Inspector-Leadership Principle}

In our sequential inspection game $\Gamma_{1}$, we will make the following special assumptions which are called the Inspector- Leadership Principle.

(i) Before the start of the game (i.e., at the zero stage), the inspector $I$ chooses and announces a test procedure which determines for which ranges of observations of relevant random variables an alarm $(A)$ is raised or not at stages $1, \ldots, n$. Formally a test procedure $\delta$ for the inspector is defined by a collection $\delta=\left(\delta_{1}, \ldots, \delta_{n}\right)$ where each $\delta_{t}, t=1, \ldots, n$, is a measurable function $\delta_{t}: X_{1} \times \ldots \times X_{t} \rightarrow\{A, \bar{A}\} . \delta_{t}$ decides $A$ (alarm) or $\bar{A}$ (no alarm) based on observations $\left(x_{1}, \ldots, x_{t}\right)$ before and at stage $t$. 


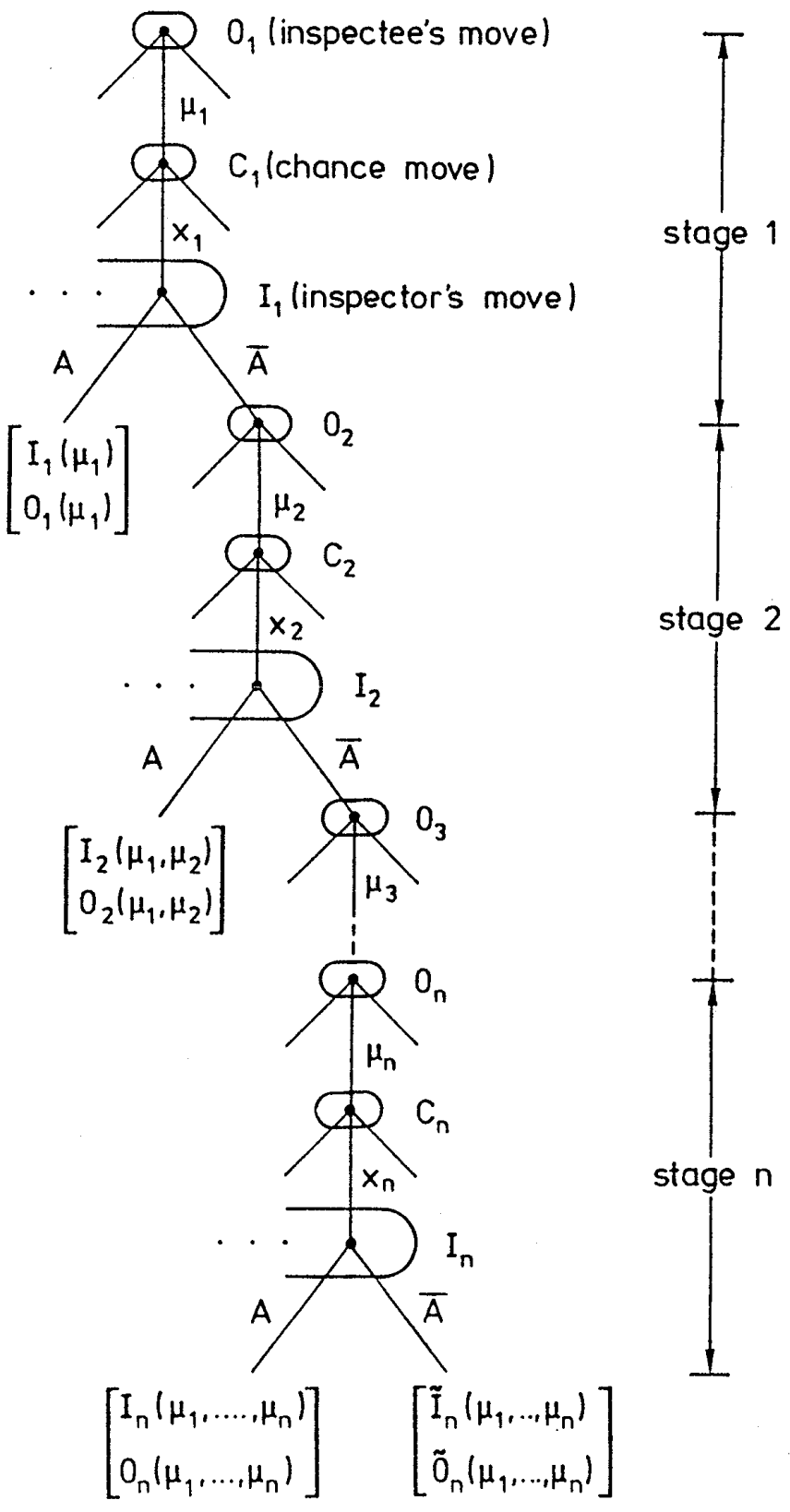

Figure 1: The extensive form of the sequential inspection game $\Gamma_{1}$ when at stage $t=1, \ldots, n 0_{t}$ is the inspectee's, $I_{t}$ the inspector's and $C_{t}$ the chance move.

(ii) Knowing the test procedure $\delta=\left(\delta_{1}, \ldots, \delta_{n}\right)$, the inspectee $O$ decides whether he will behave illegally $\left(H_{1}\right)$ or legally $\left(H_{0}\right)$. If he decides to behave illegally, he will choose an illegal vector $\mu=\left(\mu_{1}, \ldots, \mu_{n}\right)$ such that $\sum_{t} \mu_{t}=\bar{\mu}, \mu_{t} \geq 0, t=1, \ldots, n$, where $\mu_{t}$ is the size of the illegal action at stage $t=1, \ldots, n$. For convenience, we put $\mu=(0, \ldots, 0)$ in the case of legal behavior. Thereafter, the game really starts and ends either after the $i$-th stage if there is an alarm, or finally and definitely after $n$ stages. Neither player has any possibility to adjust his decision in the course of the game.

Given an illegal vector $\mu=\left(\mu_{1}, \ldots, \mu_{n}\right)$, an observation $x_{t}(t=1,2, \ldots, n)$ is drawn at stage $t$ by a chance move according to the conditional probability density function $f_{t}\left(\cdot \mid \mu_{1}, x_{1}, \ldots, \mu_{t-1}, x_{t-1}\right)$ where $\left(x_{1}, \ldots, x_{t-1}\right)$ are the observations made before stage $t$. Ba- 
sed on the observations $\left(x_{1}, \ldots, x_{t}\right)$, the inspector makes the decision according to his predetermined test procedure $\delta_{t}$, i.e., he raises an alarm if $\delta_{t}\left(x_{1}, \ldots, x_{t}\right)=A$, and does not otherwise. If an alarm is raised for the first time at stage $t$, then the game ends either with the payoffs

$$
\begin{aligned}
-a_{t}\left(\mu_{1}, \ldots, \mu_{t}\right) & \equiv I_{t}\left(\mu_{1}, \ldots, \mu_{t}\right), \\
-b_{t}\left(\mu_{1}, \ldots, \mu_{t}\right) & \equiv O_{t}\left(\mu_{1}, \ldots, \mu_{t}\right)
\end{aligned}
$$

to the inspector and the inspectee, respectively, in case of illegal behavior, or with the payoffs

$$
-e_{t} \equiv I_{t}(O, \ldots, O),-f_{t} \equiv O_{t}(O, \ldots, O)
$$

to the inspector and the inspectee, respectively, in case of legal behavior. If no alarm is raised at stage $t$, then the next stage $t+1$ will be reached. At the latest, after $n$ stages the game ends with a terminal decision of the inspector. When no alarm is raised at stage $n$, the inspector and the inspectee receive the payoffs

$$
-c\left(\mu_{1}, \ldots, \mu_{n}\right) \equiv \tilde{I}_{n}\left(\mu_{1}, \ldots, \mu_{n}\right), d\left(\mu_{1}, \ldots, \mu_{n}\right) \equiv \tilde{O}_{n}\left(\mu_{1}, \ldots, \mu_{n}\right),
$$

respectively, in case of illegal behavior, i.e., $\sum_{t} \mu_{t}=\bar{\mu}$, and the payoffs $(0,0)$ in case of legal behavior.

Given an illegal vector $\mu=\left(\mu_{1}, \ldots, \mu_{n}\right)$ of the inspectee $O$, the family of conditional probability densities $\left\{f_{t}\left(x_{t} \mid \mu_{1}, x_{1}, \ldots, \mu_{t-1}, x_{t-1}, \mu_{t}\right)\right\}_{t=1}^{n}$ generates a probability distribution $F^{\mu}\left(x_{1}, \ldots, x_{n}\right)$ over the Cartesian product $X_{1} \times \ldots \times X_{n}$. We put $F^{0}=F^{\mu}$ when $\mu=(0, \ldots, 0)$. For every $t=1, \ldots, n$, define the sets

$$
\begin{aligned}
& A_{t}^{\delta} \equiv\left\{\left(x_{1}, \ldots, x_{t}\right) \mid \delta_{t}\left(x_{1}, \ldots, x_{t}\right)=A\right\} \times X_{t+1} \times \ldots \times X_{n} \\
& \bar{A}_{t}^{\delta} \equiv\left\{\left(x_{1}, \ldots, x_{t}\right) \mid \delta_{t}\left(x_{1}, \ldots, x_{t}\right)=\bar{A}\right\} \times X_{t+1} \times \ldots \times X_{n} .
\end{aligned}
$$

$A_{t}^{\delta}$ and $\bar{A}_{t}^{\delta}$ are the sets of observations $\left(x_{1}, \ldots, x_{t}\right)$ under which the test procedure $\delta=$ $\left(\delta_{1}, \ldots, \delta_{n}\right)$ by the inspector selects $A$ (alarm) and $\bar{A}$ (no alarm), respectively, at stage $t$. We define the two probabilities

$$
\begin{gathered}
\beta_{t}\left(\delta, \mu_{1}, \ldots, \mu_{t}\right)=P^{\mu}\left(\bar{A}_{t}^{\delta} \mid \bar{A}_{1}^{\delta} \cap \ldots \cap \bar{A}_{t-1}^{\delta}\right) \\
\alpha_{t}(\delta)=P^{0}\left(A_{t}^{\delta} \mid \bar{A}_{1}^{\delta} \cap \ldots \cap \bar{A}_{t-1}^{\delta}\right)
\end{gathered}
$$

where $P^{\mu}(\cdot \mid \cdot)$ and $P^{0}(\cdot \mid \cdot)$ are the conditional probabilities induced by the distributions $F^{\mu}$ and $F^{0}$, respectively. Under the condition that the game reaches stage $t, \beta_{t}\left(\delta, \mu_{1}, \ldots, \mu_{t}\right)$ represents the 'no detection' probability at stage $t$ and $\alpha_{t}(\delta)$ represents the 'false alarm' probability at stage $t$.

With the inspector-leadership principle described by (i) and (ii), the sequential inspection game $\Gamma_{1}$ in the first section can be transformed into a simpler game whose extensive form is sketched in Figure 2; we call it the sequential inspector-leadership game, denoted by $\Gamma_{2}$.

In what follows, we will analyze the sequential inspector-leadership game $\Gamma_{2}$. A (pure) strategy for the inspector in $\Gamma_{2}$ is defined to be a test procedure $\delta=\left(\delta_{1}, \ldots, \delta_{n}\right)$. A (pure) strategy for the inspectee in $\Gamma_{2}$ is defined to be $\gamma=\left(\gamma_{1}, \gamma_{2}\right)$ where $\gamma_{1}$ assigns to every $\delta$ either $H_{0}$ or $H_{1}$ and $\gamma_{2}$ to every $\delta$ a diversion vector 


$$
\mu=\left(\mu_{1}, \ldots, \mu_{n}\right), \sum_{t=1}^{n} \mu_{t}=\bar{\mu}, \mu_{t} \geq 0, t=1, \ldots, n
$$

Given a strategy pair $(\delta, \gamma)$, the expected payoffs for the inspector and the inspectee, denoted by $E g_{1}(\delta, \gamma)$ and $E g_{2}(\delta, \gamma)$, respectively, are defined in a usual manner. Also, the conditional expected payoff for the inspectee given that the inspector selects $\delta$ and the inspectee selects $H_{1}$ can be defined. It is denoted by $E g_{2}\left(\gamma \mid \delta, H_{1}\right)$.

The standard solution concept of a non-cooperative game is the Nash equilibrium point. However, in a game with sequential structures, it has been commonly discussed that every Nash equilibrium point is not reasonable as a non-cooperative solution of the game. Some Nash equilibrium points may be supported by players' incredible threats which are not consistent with their payoff maximizing behavior. In order to eliminate such a difficulty of the Nash equilibrium point, we will employ a stronger notion of a subgame perfect equilibrium point to analyze the sequential inspector-leadership game $\Gamma_{2}$.

We can now define a subgame perfect equilibrium point of the sequential inspector leadership game $\Gamma_{2}$.

\section{Definition 3.1}

A strategy pair $\left(\delta^{*}, \gamma^{*}\right)$ is a subgame perfect equilibrium point of $\Gamma_{2}$ if and only if

(1) $E g_{1}\left(\delta^{*}, \gamma^{*}\right) \geq E g_{1}\left(\delta, \gamma^{*}\right)$ for all $\delta$,

(2) $E g_{2}\left(\delta, \gamma^{*}\right) \geq E g_{2}(\delta, \gamma)$ for all $\delta$ and all $\gamma$,

(3) $E g_{2}\left(\gamma^{*} \mid \delta, H_{1}\right) \geq E g_{2}\left(\gamma \mid \delta, H_{1}\right)$ for all $\delta$ and all $\gamma$.

The next lemma explicitly shows the expected payoffs for the inspector and the inspectee in $\Gamma_{2}$. The proof of the lemma is left to readers.

\section{Lemma 3.1}

Given a strategy pair $(\delta, \gamma)$ with $\delta=\left(\delta_{1}, \ldots, \delta_{n}\right), \gamma=\left(\gamma_{1}, \gamma_{2}\right)$ and $\gamma_{2}(\delta)=\left(\mu_{1}, \ldots, \mu_{n}\right)$, the expected payoffs for the inspector and the inspectee in $\Gamma_{2}$ are given as follows:

$$
\begin{aligned}
& E g_{1}(\delta, \gamma)= \begin{cases}-\sum_{t=1}^{n} a_{t}\left(1-\beta_{t}\right) \prod_{i=1}^{t-1} \beta_{i}-c \prod_{t=1}^{n} \beta_{t} & \text { if } \gamma_{1}(\delta)=H_{1} \\
-\sum_{t=1}^{n} e_{t} \alpha_{t} \prod_{i=1}^{t-1}\left(1-\alpha_{i}\right) & \text { if } \gamma_{1}(\delta)=H_{0}\end{cases} \\
& E g_{2}(\delta, \gamma)= \begin{cases}-\sum_{t=1}^{n} b_{t}\left(1-\beta_{t}\right) \prod_{i=1}^{t-1} \beta_{i}+d \prod_{t=1}^{n} \beta_{t} & \text { if } \gamma_{1}(\delta)=H_{1} \\
-\sum_{t=1}^{n} f_{t} \alpha_{t} \prod_{i=1}^{t-1}\left(1-\alpha_{i}\right) & \text { if } \gamma_{1}(\delta)=H_{0}\end{cases}
\end{aligned}
$$

where $a_{t}=a_{t}\left(\mu_{1}, \ldots, \mu_{t}\right), b_{t}=b_{t}\left(\mu_{1}, \ldots, \mu_{t}\right), \beta_{t}=\beta_{t}\left(\delta, \mu_{1}, \ldots, \mu_{t}\right)$ and $a_{t}=\alpha_{t}(\delta)$ for $t=$ $1, \ldots, n$.

The next proposition characterizes a subgame perfect equilibrium point of the sequential inspector-leadership game $\Gamma_{2}$. 


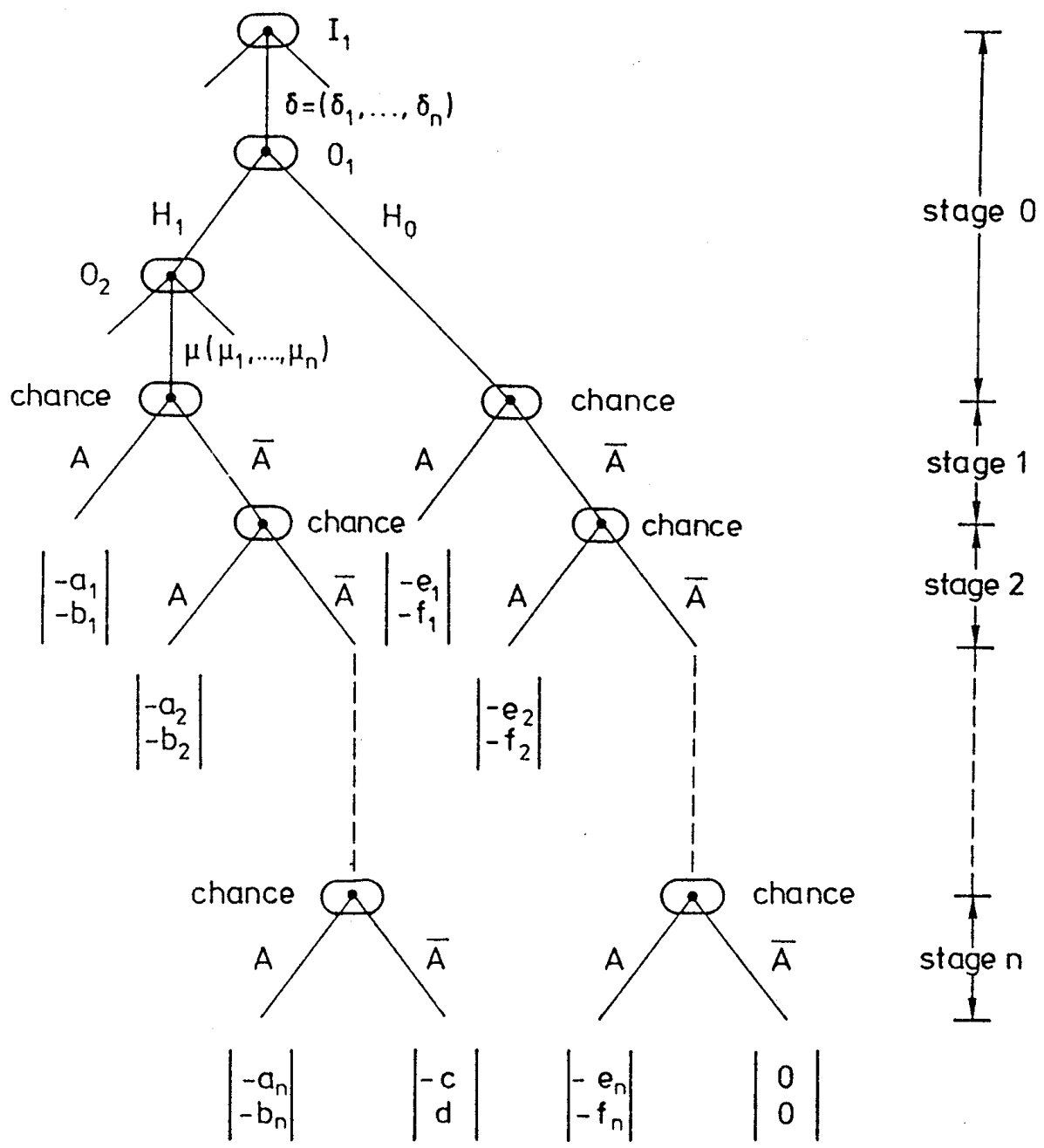

Figure 2: A sequential inspector-leadership game $\Gamma_{2} . \delta$ is a test procedure of the inspector, $H_{0}\left(H_{1}\right.$, resp.) is legal (illegal, resp.) behavior of the inspectee.' $A$ ' means alarm and ' $\bar{A}$ ' no alarm. The probability for alarm at stage $t$ is

$$
\begin{cases}1-\beta_{t}\left(\delta, \mu_{1}, \ldots, \mu_{t}\right) & \text { if } H_{1} \\ \alpha_{t}(\delta) & \text { if } H_{0}\end{cases}
$$

Proposition 3.2

A subgame perfect equilibrium point $\left(\delta^{*}, \gamma^{*}\right)$ of $\Gamma_{2}, \delta^{*}=\left(\delta_{1}^{*}, \ldots, \delta_{n}^{*}\right), \gamma^{*}=\left(\gamma_{1}^{*}, \gamma_{2}^{*}\right)$, satisfies the following conditions:

(1) For every $\delta, \gamma_{2}^{*}(\delta)=\left(\mu_{1}^{*}, \ldots, \mu_{n}^{*}\right)$ is a solution of

$$
\max _{\substack{\mu=\left(\mu_{1}, \ldots, \mu_{n}\right) \\ \sum_{t=1}^{n} \mu_{t}=\tilde{\mu}}}\left[-\sum_{t=1}^{n} b_{t}\left(1-\beta_{t}\right) \prod_{i=1}^{t-1} \beta_{i}+d \prod_{t=1}^{n} \beta_{t}\right] \text {. }
$$

We denote this maximum value by $M(\delta)$.

(2) For every $\delta, \gamma_{1}^{*}(\delta)$ is equal to

$$
\begin{aligned}
& H_{0} \quad \text { if } \quad M(\delta)<-\sum_{t=1}^{n} f_{t} \alpha_{t} \prod_{i=1}^{t-1}\left(1-\alpha_{i}\right) \\
& H_{1} \quad \text { if } \quad M(\delta)>-\sum_{t=1}^{n} f_{t} \alpha_{t} \prod_{i=1}^{t-1}\left(1-\alpha_{i}\right) .
\end{aligned}
$$


(Note that here we leave open the case

$$
\left.M(\delta)=-\sum_{t=1}^{n} f_{t} \alpha_{t} \prod_{i=1}^{t-1}\left(1-\alpha_{i}\right) .\right)
$$

(3) $\delta^{*}$ is a solution of $\sup _{\delta} I(\delta)$ where

$$
I(\delta)= \begin{cases}-\sum_{t=1}^{n} e_{t} \alpha_{t} \prod_{i=1}^{t-1}\left(1-\alpha_{i}\right) & \text { if } \gamma_{1}^{*}(\delta)=H_{0} \\ -\sum_{t=1}^{n} a_{t}\left(1-\beta_{t}\right) \prod_{i=1}^{t-1} \beta_{i}-c \prod_{t=1}^{n} \beta_{t} & \text { if } \gamma_{1}^{*}(\delta)=H_{1}\end{cases}
$$

where $a_{t}=a_{t}\left(\mu_{1}^{*}, \ldots, \mu_{t}^{*}\right)$ and $\beta_{t}=\beta_{t}\left(\delta ; \mu_{1}^{*}, \ldots, \mu_{t}^{*}\right)$ for $t=1, \ldots, n$.

\section{Proof}

We can prove the proposition by the 'backward induction' argument in the theory of extensive games. First, from Lemma 3.1, the conditional expected payoff $E g_{2}\left(\gamma \mid \delta, H_{1}\right)$ for the inspectee $O$ when the inspector selects a test procedure $\delta=\left(\delta_{1}, \ldots, \delta_{n}\right)$ and he selects illegal behavior $H_{1}$ is given by

$$
E g_{2}\left(\gamma \mid \delta, H_{1}\right)=-\sum_{t=1}^{n} b_{t}\left(1-\beta_{t}\right) \prod_{i=1}^{t-1} \beta_{i}+d \prod_{t=1}^{n} \beta_{t}
$$

Then, Definition 3.1.(3) is equivalent to (3.3).

Secondly, when $\gamma_{2}^{*}(\delta)$ satisfies (3.3), we can show that Definition 3.1.(2) and (3.2) imply (3.4). Finally, we can consider the optimal test procedure $\delta^{*}=\left(\delta_{1}^{*}, \ldots, \delta_{n}^{*}\right)$ of the inspector. When he selects a test procedure $\delta$, he obtains the expected payoff $I(\delta)$ given by (3.5), depending on whether $\gamma_{1}^{*}(\delta)=H_{0}$ or $H_{1}$. Therefore, Definition 3.1.(1) implies that $\delta^{*}$ is a solution of $\sup _{\delta} I(\delta)$.

As already mentioned, in this proposition we did not determine $\gamma_{1}^{*}(\delta)$ for the case $M(\delta)=-\sum_{t} f_{t} \alpha_{t} \prod_{i}\left(1-\alpha_{i}\right)$. In the following two sections, we consider two special forms of the payoff parameters where just this case becomes the important one.

\section{An Analysis of the Sequential Inspector-Leadership Game-A Finite Time Horizon Case}

In this section, we will consider under what conditions the sequential inspector-leadership game $\Gamma_{2}$ given in the last section can be equivalently reduced to the non-sequential inspector-leadership game discussed by Avenhaus and Okada [2].

Let us assume the very special situation that the payoffs for the inspector and the inspectee are independent of the stage number, and, furthermore, that they are independent of the diversion vector $\mu=\left(\mu_{1}, \ldots, \mu_{n}\right)$ in case of illegal behavior:

\section{Assumption 4.1}

There exist positive constants, $a, b, c, d, e, f$ such that

$$
\begin{gathered}
a=a_{t}\left(\mu_{1}, \ldots, \mu_{t}\right), b=b_{t}\left(\mu_{1}, \ldots, \mu_{t}\right) \\
c=c\left(\mu_{1}, \ldots, \mu_{n}\right), d=d\left(\mu_{1}, \ldots, \mu_{n}\right) \\
e=e_{t}, f=f_{t}
\end{gathered}
$$

for every $t=1, \ldots, n$ and $\mu=\left(\mu_{1}, \ldots, \mu_{n}\right)$ with $\sum_{t} \mu_{t}=\bar{\mu}, \mu_{t} \geq 0(t=1, \ldots, n)$. We assume 
that

$$
e<a<c, f<b .
$$

The next lemma shows that under Assumption 4.1 the expected payoffs of the inspector and the inspectee can be simply represented in terms of global 'non-detection' probability and 'false alarm' probability. The proof of the lemma is left to readers.

\section{Lemma 4.2}

Under Assumption 4.1, the expected payoffs for the inspector and the inspectee in $\Gamma_{2}$ are given as follows:

$$
\begin{aligned}
& E g_{1}(\delta, \gamma)= \begin{cases}-a(1-\beta(\delta, \mu))-c \beta(\delta, \mu) & \text { if } \gamma_{1}(\delta)=H_{1} \\
-e \alpha(\delta) & \text { if } \gamma_{1}(\delta)=H_{0}\end{cases} \\
& E g_{2}(\delta, \gamma)= \begin{cases}-b(1-\beta(\delta, \mu))+d \beta(\delta, \mu) & \text { if } \gamma_{1}(\delta)=H_{1} \\
-f \alpha(\delta) & \text { if } \gamma_{1}(\delta)=H_{0}\end{cases}
\end{aligned}
$$

where $\beta(\delta, \mu)=\prod_{t} \beta_{t}\left(\delta_{t} ; \mu_{1}, \ldots, \mu_{t}\right)$ and $\alpha(\delta)=1-\prod_{t}\left(1-\alpha_{t}(\delta)\right)$ are the overall nondetection and false alarm probabilities.

By Lemma 4.2, we can reduce the sequential inspector-leadership game $\Gamma_{2}$ into the non-sequential inspector-leadership game $\Gamma_{3}$ the extensive form of which is given in Figure 3. With the help of this reduction, we can investigate a subgame perfect equilibriu point of the sequential inspector-leadership game $\Gamma_{2}$.

Let $\delta=\left(\delta_{1}, \ldots, \delta_{n}\right)$ be a pure strategy, i.e., a test procedure for the inspector and let $\gamma=\left(\gamma_{1}, \gamma_{2}\right)$ be a pure strategy for the inspectee. Given $\alpha \in[0,1]$, define the set

$$
\Delta_{\alpha}=\left\{\delta=\left(\delta_{1}, \ldots, \delta_{n}\right) \mid \alpha(\delta) \equiv 1-\prod_{t=1}^{n}\left(1-\alpha_{t}(\delta)\right)=\alpha\right\}
$$

and

$$
\begin{gathered}
\beta(\delta)=\max _{\mu} \beta(\delta, \mu) \\
\beta(\alpha)=\min _{\delta \in \Delta_{\alpha}} \max _{\mu} \beta(\delta, \mu)
\end{gathered}
$$

where $\beta(\delta, \mu)=\prod_{t} \beta_{t}\left(\delta, \mu_{1}, \ldots, \mu_{t}\right), \mu=\left(\mu_{1}, \ldots, \mu_{n}\right)$.

A subgame perfect equilibrium point of the non-sequential inspector-leadership game $\Gamma_{3}$ is characterized in [2]. From this result we obtain the following:

Theorem 4.3

A pure strategy combination $\left(\delta^{*}, \gamma^{*}\right), \delta^{*}=\left(\delta_{1}^{*}, \ldots, \delta_{n}^{*}\right), \gamma^{*}=\left(\gamma_{1}^{*}, \gamma_{2}^{*}\right)$ of the non-sequential inspector- leadership game $\Gamma_{3}$ is a subgame perfect equilibrium point of $\Gamma_{3}$, if and only if the following conditions are satisfied:

(1) The 'false alarm' probability

$$
\alpha^{*}=\alpha\left(\delta^{*}\right)=1-\prod_{t=1}^{n}\left(1-\alpha_{t}\left(\delta^{*}\right)\right)
$$


of $\delta^{*}$ is a solution of the equation

$$
-b+(b+d) \beta(\alpha)+f \alpha=0
$$

where $\beta(\alpha)$ is given by (4.5).

(2) $\delta^{*}=\left(\delta_{1}^{*}, \ldots, \delta_{n}^{*}\right)$ is a solution of the optimization problem

$$
\min _{\delta \in \Delta_{\alpha}} \max _{\mu} \beta(\delta, \mu)=\min _{\delta \in \Delta_{\alpha}} \max _{\mu} \prod_{t=1}^{n} \beta_{t}\left(\delta, \mu_{1}, \ldots, \mu_{t}\right) .
$$

(3) For every $\delta=\left(\delta_{1}, \ldots, \delta_{n}\right)$

$$
\gamma_{1}^{*}(\delta)= \begin{cases}H_{0} & \text { if } h(\delta) \leq 0 \\ H_{1} & \text { if } h(\delta)>0\end{cases}
$$

where

$$
h(\delta) \equiv-b+(b+d) \beta(\delta)+f \alpha(\delta),
$$

and $\beta(\delta)$ is given by (4.4).

(4) For every $\delta=\left(\delta_{1}, \ldots, \delta_{n}\right), \gamma_{2}^{*}(\delta)$ is a solution of

$$
\max _{\mu} \beta(\delta, \mu)=\max _{\mu} \prod_{t=1}^{n} \beta_{t}\left(\delta, \mu_{1}, \ldots, \mu_{t}\right) .
$$

Under Assumption 4.1 the stage number $t(t=1, \ldots, n)$ has no influence on players' payoffs when the game stops at stage $t$ with an alarm by the inspector: The early detection does not make the inspector better off. Furthermore, the later the inspector makes a decision, the more information he can obtain. Intuitively, these observations suggest that in the sequential inspector-leadership game $\Gamma_{2}$ the inspector need not raise an alarm until the last stage.

\section{An Analysis of the Sequential Inspector-Leadership Game: An Infinite Time Horizon Case}

Let us consider the sequential inspector-leadership game of the third section with infinitely many stages, and let us assume that the payoffs to both players at each stage depend on the stage number as follows:

\section{Assumption 5.1}

There exist positive constants $\nu_{1}, \nu_{2}, \lambda_{1}, \lambda_{2}$ such that the payoffs to both players are given by

$$
\begin{gathered}
a_{t}\left(\mu_{1}, \ldots, \mu_{t}\right)=a \exp \left(\nu_{1} t\right), b_{t}\left(\mu_{1}, \ldots, \mu_{t}\right)=b \exp \left(-\nu_{2} t\right), \\
e_{t}=e \exp \left(-\lambda_{1} t\right), f_{t}=f \exp \left(-\lambda_{2} \cdot t\right)
\end{gathered}
$$

for every $t=1,2, \ldots$ and every $\mu=\left(\mu_{1}, \mu_{2}, \ldots\right)$ where $0<e<a$ and $0<f<b$.

Assumption 5.1 shows that the losses, $e_{t}$ and $f_{t}$, caused by false alarm are exponentially decreasing with respect to $t$. An earlier false alarm imposes more damages on both players. Secondly, the two players have opposite time preferences with respect to the detection of illegal behavior. The inspector prefers earlier detection, while the inspectee prefers later detection. 


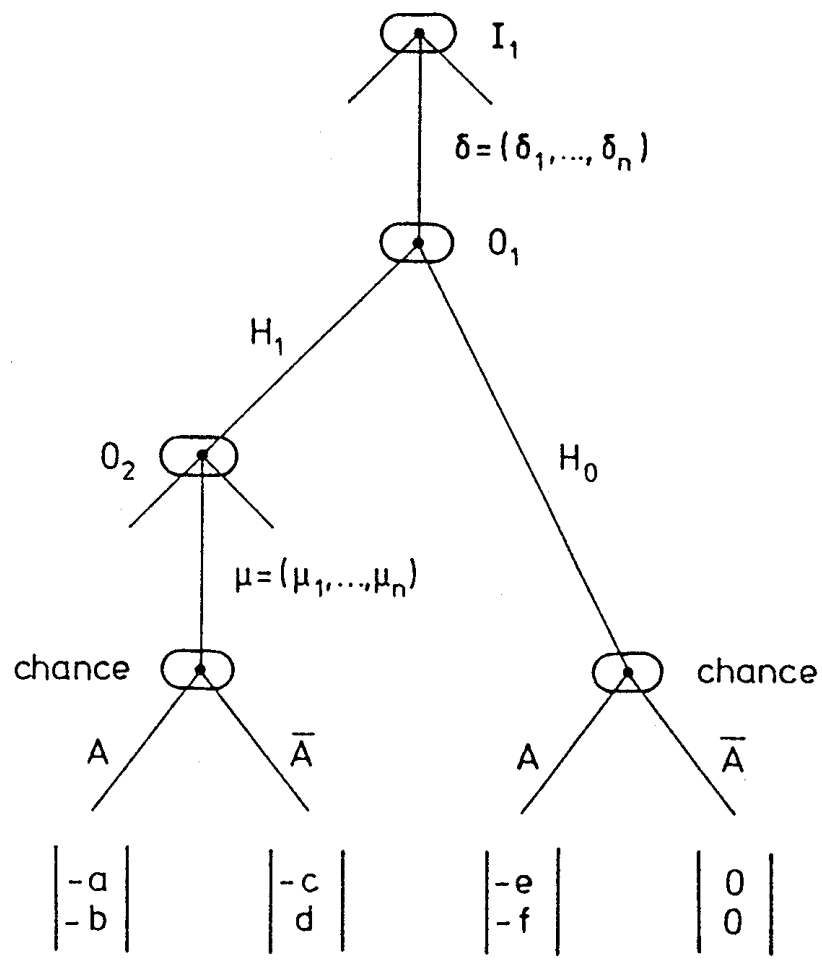

Figure 3: The non-sequential inspector-leadership game $\Gamma_{3}$ to which the sequential inspector-leadership game $\Gamma_{2}$ can be equivalently reduced. The probability for alarm is given by

$$
\begin{aligned}
1-\beta(\delta, \mu) & =1-\prod_{t=1}^{n} \beta_{t}\left(\delta, \mu_{1}, \ldots, \mu_{t}\right) \text { if } H_{1} \\
\alpha(\delta) & =1-\prod_{t=1}^{n}\left(1-\alpha_{t}(\delta)\right) \quad \text { if } H_{0}
\end{aligned}
$$

The sequential inspector-leadership game with infinitely many stages is denoted by $\Gamma_{4}$. As in the game $\Gamma_{2}$, we can define a test procedure $\delta=\left(\delta_{1}, \delta_{2}, \ldots\right)$ of the inspector and a diversion strategy $\mu=\left(\mu_{1}, \mu_{2}, \ldots\right)$ of the inspectee with $\Sigma_{t} \mu_{t}=\bar{\mu}$ : In what follows, we use the same notations as in the third section whenever no confusion arises. For a strategy pair $(\delta, \gamma)$, we can define the 'false alarm' probability at stage $t$, denoted by $\alpha_{t}(\delta)$, and the 'no detection' probability at stage $t$, denoted by $\beta_{t}(\delta, \mu)$.

\section{Lemma 5.2}

Under Assumption 5.1 the expected payoffs to the inspector and the inspectee in $\Gamma_{4}$ are

$$
E g_{1}(\delta, \gamma)= \begin{cases}-a \sum_{t=1}^{\infty} \exp \left(\nu_{1} t\right)\left(1-\beta_{t}\right) \prod_{s=1}^{t-1} \beta_{s} & \\ =-a F_{1}\left(\nu_{1} ; \delta, \mu\right) & \text { if } \gamma_{1}(\delta)=H_{1} \\ -e \sum_{t=1}^{\infty} \exp \left(-\lambda_{1} t\right) \alpha_{t} \prod_{s=1}^{t-1}\left(1-\alpha_{s}\right) & \\ =-e F_{0}\left(-\lambda_{1} ; \delta\right) & \text { if } \gamma_{1}(\delta)=H_{0}\end{cases}
$$




$$
E g_{2}(\delta, \gamma)=\left\{\begin{array}{cc}
-b \sum_{t=1}^{\infty} \exp \left(-\nu_{2} t\right)\left(1-\beta_{t}\right) \prod_{s=1}^{t-1} \beta_{s} & \\
=-b F_{1}\left(-\nu_{2} ; \delta, \mu\right) & \text { if } \gamma_{1}(\delta)=H_{1} \\
-f \sum_{t=1}^{\infty} \exp \left(-\lambda_{2} t\right) \alpha_{t} \prod_{s=1}^{t-1}\left(1-\alpha_{s}\right) & \\
=-f F_{0}\left(-\lambda_{2} ; \delta\right) & \text { if } \gamma_{1}(\delta)=H_{0}
\end{array}\right.
$$

where $F_{1}(\cdot ; \delta, \mu)$ and $F_{0}(\cdot ; \delta)$ are the moment generating functions of the stages (random variables) that an alarm is raised in the cases of illegal behavior and legal behavior, respectively.

\section{Proof}

By Assumption 5.1 we have

$$
E g_{1}(\delta, \gamma)=-a \sum_{t=1}^{\infty} \exp \left(\nu_{1} t\right) \operatorname{Prob}(\text { alarm at } t \mid \delta, \mu)
$$

where Prob(alarm at $t \mid \delta, \mu)$ is the probability of alarm at stage $t$ if the inspector selects a test procedure $\delta$ and the inspectee selects the strategy $\gamma=\left(\gamma_{1}, \gamma_{2}\right)$ with $\gamma_{1}(\delta)=H_{1}$ and $\gamma_{2}(\delta)=\mu$. Since

$$
\operatorname{Prob}(\text { alarm at } t \mid \delta, \mu)=\left(1-\beta_{t}\right) \prod_{s=1}^{t-1} \beta_{s}
$$

we can prove the first equality in case of $\gamma_{1}(\delta)=H_{1}$ in (5.1). The second equality in that case is clear from the definition of the moment generating function $F_{1}(\cdot ; \delta, \mu)$ of the false alarm stage. Similar calculations lead to other equalities in (5.1) and (5.2).

From the well-known property of the moment generating function we can apply the Taylor expansion to (5.1) and (5.2). For example, we have

$$
\begin{gathered}
F_{1}\left(-\nu_{2} ; \delta, \mu\right)=F_{1}(0 ; \delta, \mu)+\left(-\nu_{2}\right) \cdot F_{1}^{(1)}(0 ; \delta, \mu)+\ldots \\
+\left(-\nu_{2}\right)^{n} / n ! \cdot F_{1}^{(n)}(0 ; \delta, \mu)+\ldots
\end{gathered}
$$

for small values of $\nu_{2}$ where $F_{1}^{(n)}(0 ; \delta, \mu)$ is the $n$-th order derivative at $\theta=0$ of $F_{1}(\theta ; \delta, \mu)$. The first term of $(5.3)$ is $F_{1}(0 ; \delta, \mu)=1$; the second of $(5.3)$, given explicitly by

$$
F_{1}^{(1)}(0 ; \delta ; \mu)=\sum_{t=1}^{\infty} t\left(1-\beta_{t}\right) \prod_{s=1}^{t-1} \beta_{s}
$$

is the average run length of the game in case of illegal behavior.

We denote this by $L_{1}(\delta, \mu)$. Similarly, we denote by $L_{0}(\delta)$ the average run length of the game in case of legal behavior which is given by

$$
F_{0}^{(1)}(0 ; \delta)=\sum_{t=1}^{\infty} t \alpha_{t} \prod_{s=1}^{t-1}\left(1-\alpha_{s}\right) .
$$

In what follows we will employ the first-order approximations of the expected payoffs of the inspector and the inspectee.

\section{Assumption 5.3}

For a strategy pair $(\delta, \gamma)$, the expected payoffs to the inspector and the inspectee are 


$$
E g_{1}(\delta, \gamma)= \begin{cases}-a\left(1+\nu_{1} L_{1}(\delta, \mu)\right) & \text { if } \gamma_{1}(\delta)=H_{1} \\ -e\left(1-\lambda_{1} L_{0}(\delta)\right) & \text { if } \gamma_{1}(\delta)=H_{0}\end{cases}
$$

and

$$
E g_{2}(\delta, \gamma)= \begin{cases}-b\left(1-\nu_{2} L_{1}(\delta, \mu)\right) & \text { if } \gamma_{1}(\delta)=H_{1} \\ -f\left(1-\lambda_{2} L_{0}(\delta)\right) & \text { if } \gamma_{1}(\delta)=H_{0}\end{cases}
$$

Furthermore, we will use the following assumptions on the average run lengths of the tests under consideration which are satisfied for commonly used sequential tests.

\section{Assumption 5.4}

Let $\Delta$ be the set of test procedures $\delta=\left(\delta_{1}, \delta_{2} \ldots\right)$ of the inspector. For a given value $L_{0} \geq 1$, we define the set $\Delta_{L_{0}}=\left\{\delta \in \Delta \mid L_{0}(\delta)=L_{0}\right\}$.

(1) For every $L_{0} \geq 1$, the minimax problem

$$
\min _{\delta \in \Delta_{L_{0}}} \max _{\mu} L_{1}(\delta, \mu)
$$

has a solution. We denote by $L_{1}(\delta)$ the maximum and by $L_{1}\left(L_{0}\right)$ the minimax value of $L_{1}(\delta, \mu)$, i.e.,

$$
\begin{gathered}
L_{1}(\delta)=\max _{\mu} L_{1}(\delta, \mu) \\
L_{1}\left(L_{0}\right)=\min _{\delta \in \Delta_{L_{0}}} \max _{\mu} L_{1}(\delta, \mu) .
\end{gathered}
$$

(2) The function $L_{1}\left(L_{0}\right)$ is a continuous and monotonically increasing function on $[1, \infty)$ with $L_{1}(1)=1$.

(3) There is a unique solution of the equation

$$
-b\left(1-\nu_{2} L_{1}\left(L_{0}\right)\right)+f\left(1-\lambda_{2} L_{0}\right)=0
$$

A very simple example for a sequential test procedure with properties (2) is given in the Annex. With the assumptions 5.3 and 5.4 we will characterize a subgame perfect equilibrium point of the game $\Gamma_{4}$.

\section{Theorem 5.5}

Under Assumptions 5.3 and 5.4, a pure strategy combination $\left(\delta^{*}, \gamma^{*}\right)$ of the sequential inspector-leadership game $\Gamma_{4}$ is a subgame perfect equilibrium point of $\Gamma_{4}$ if and only if the following conditions are satisfied:

(1) The average run length $L_{0}\left(\delta^{*}\right)$ under $H_{0}$ is the solution $L_{0}^{*}$ of equation (5.7) where $L_{1}\left(L_{0}\right)$ is given by $(5.6)$.

(2) The test procedure $\delta^{*}$ of the inspector is a solution of the minimax problem

$$
\min _{\delta \in \Delta_{L_{0}^{*}}} \max _{\mu} L_{1}(\delta, \mu)
$$


(3) For every $\delta \in \Delta$, the inspectee behaves according to

$$
\gamma_{1}^{*}(\delta)= \begin{cases}H_{0} & \text { if } h(\delta) \leq 0 \\ H_{1} & \text { if } h(\delta)>0\end{cases}
$$

where $h(\delta)$ is given by

$$
h(\delta) \equiv-b\left(1-\nu_{2} L_{1}(\delta)\right)+f\left(1-\lambda_{2} L_{0}(\delta)\right) .
$$

(4) For every $\delta \in \Delta$, the illegal strategy $\gamma_{2}^{*}(\delta)$ of the inspectee is a solution of

$$
L_{1}(\delta)=\max _{\mu} L_{1}(\delta, \mu)
$$

\section{Proof}

We will prove the theorem with the help of the 'backward induction' argument frequently used in the theory of extensive games. Let $\left(\delta^{*}, \gamma^{*}\right)$ be a subgame perfect equilibrium point of $\Gamma_{4}$.

First, for every $\delta \in \Delta, \gamma_{2}^{*}(\delta)$ is a solution of

$$
\max _{\mu}-b\left(1-\nu_{2} L_{1}(\delta, \mu)\right)
$$

from Assumption 5.3. Since $b, \nu_{2}>0, \gamma_{2}^{*}(\delta)$ is a solution of the maximization $L_{1}(\delta)=$ $\max L_{1}(\delta, \mu)$. This proves (4) in the theorem.

Secondly, for every $\delta \in \Delta, \gamma_{1}^{*}(\delta)$ is a solution of $\max E g_{2}(\delta, \gamma)$ where $\gamma_{2}(\delta)$ is given above. From (5.5) we can see that

$$
\gamma_{1}^{*}(\delta)= \begin{cases}H_{0} & \text { if } h(\delta)<0 \\ H_{0} \text { or } H_{1} & \text { if } h(\delta)=0 \\ H_{1} & \text { if } h(\delta)>0\end{cases}
$$

where $h(\delta)$ is given by (5.9). This leads to (3).

Thirdly, $\delta^{*}$ is a solution of $\max _{\delta} E g_{1}\left(\delta, \gamma^{*}\right)$ where $\gamma^{*}=\left(\gamma_{1}^{*}, \gamma_{2}^{*}\right)$ is given by (3) and (4). Since we have $\Delta=\cup_{L_{0} \geq 1} \Delta_{L_{0}}$, we can decompose this maximization problem as follows:

$$
\max _{L_{0} \geq 1} \max _{\delta \in \Delta_{L_{0}}} E g_{1}\left(\delta, \gamma^{*}\right)
$$
$\delta \in \Delta_{L_{0}}$

We first analyze the subproblem $\max _{\delta \in \Delta_{L_{0}}} E g_{1}\left(\delta, \gamma^{*}\right)$. From (5.4) and (5.8) we have for

$$
E g_{1}\left(\delta, \gamma^{*}\right)= \begin{cases}-e\left(1-\lambda_{1} L_{0}\right) & \text { if } h(\delta)<0 \\ -a\left(1+\nu_{1} L_{1}(\delta)\right) & \text { if } h(\delta)>0\end{cases}
$$

Let $L_{0}^{*}$ be the solution of (5.7). Geometrically, $L_{0}^{*}$ is given by the intersection of the curve $L_{1}=L_{1}\left(L_{0}\right)$ and the line $\left(1 / \nu_{2}\right)\left(1-(f / b)\left(1-\lambda_{2} L_{0}\right)\right)$, see Figure 4a. Since, according to Assumption 5.4, $L_{1}=L_{1}\left(L_{0}\right)$ is monotonically increasing, we have

$$
\begin{aligned}
& h(\delta)<0 \text { if } 1 \leq L_{0}(\delta)<L_{0}^{*} \text { and } L_{1}(\delta)<\frac{1}{\nu_{2}}\left(1-\frac{f}{b}\left(1-\lambda_{2} L_{0}\right)\right) \\
& h(\delta)=0 \text { if } 1 \leq L_{0}(\delta) \leq L_{0}^{*} \text { and } L_{1}(\delta)=\frac{1}{\nu_{2}}\left(1-\frac{f}{b}\left(1-\lambda_{2} L_{0}\right)\right) \\
& h(\delta)>0 \text { otherwise. }
\end{aligned}
$$




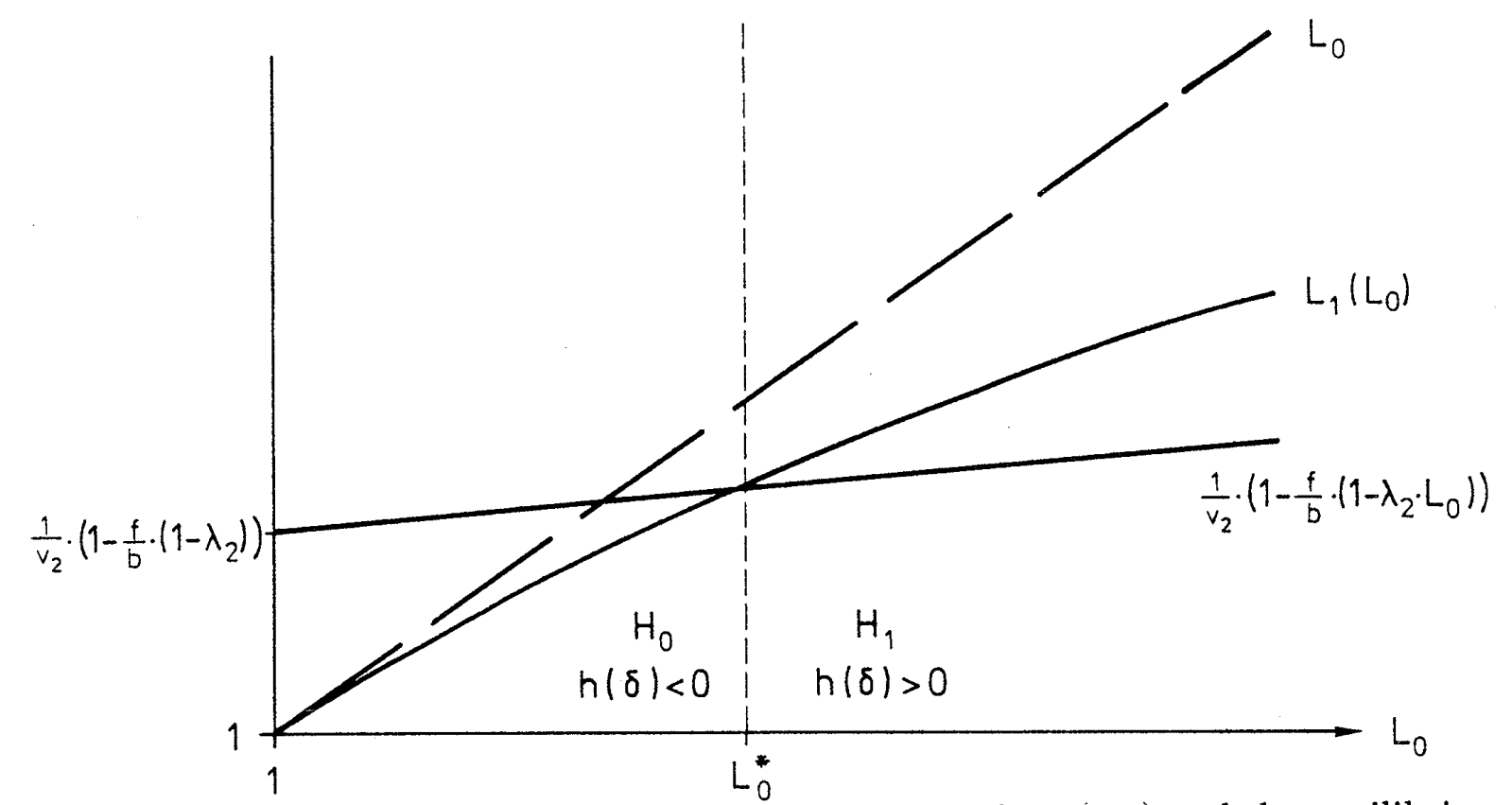

Figure 4a: Graphical representation of the solution of eq. (5.7) and the equilibrium strategy $\gamma_{1}^{*}$ of the inspectee.

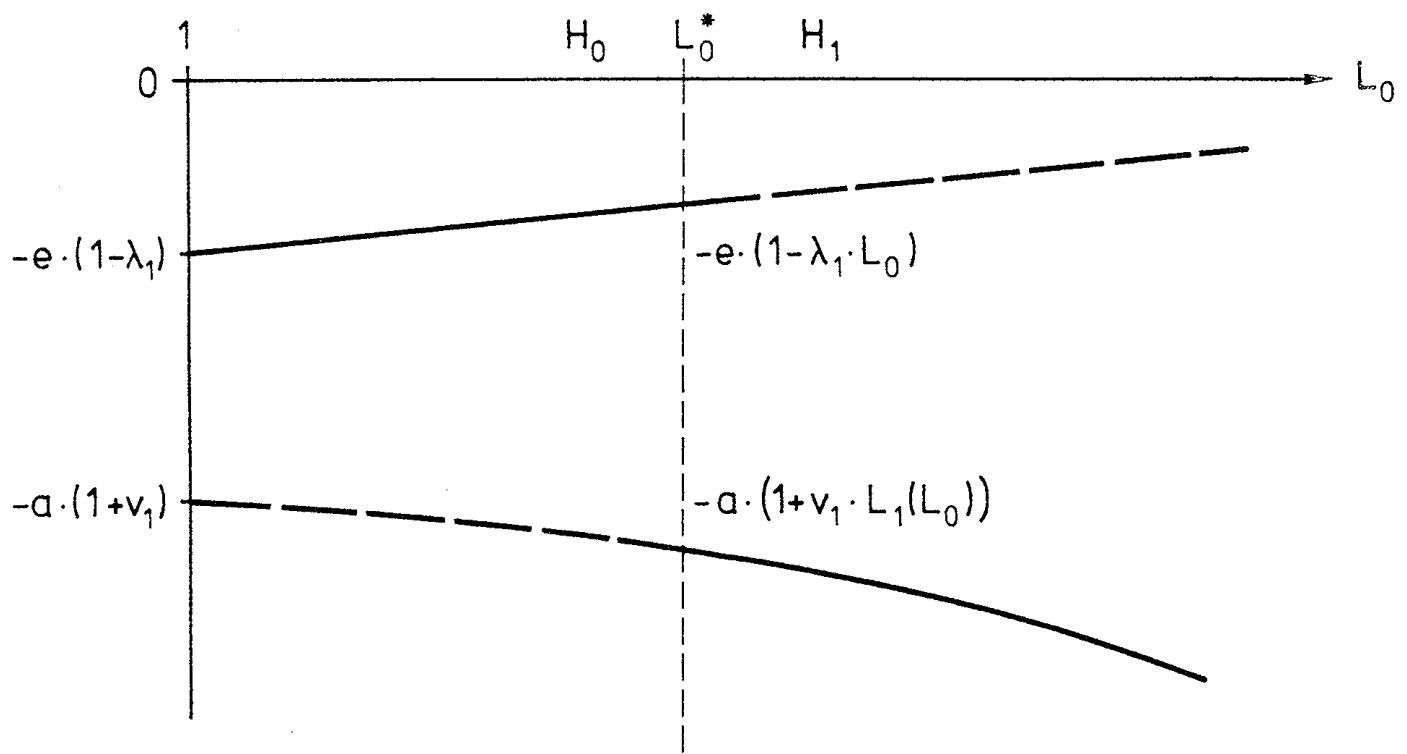

Figure 4b: Graphical representation of the inspector's payoff as a function of $L_{0}$, according to (5.13).

From (5.11) and (5.12), for every $L_{0}$ with $L_{0}>L_{0}^{*}$ and every $\delta \in \Delta_{L_{0}}$, we have $E g_{1}\left(\delta, \gamma^{*}\right)=-a\left(1+\nu_{1} L_{1}(\delta)\right)$. Therefore, for every $L_{0}>L_{0}^{*}$, the subproblem $\max E g_{1}\left(\delta, \gamma^{*}\right)$ can be equivalently reduced to $\min L_{1}(\delta)$, since $a, \nu_{1}>0$ and its solution is $L_{1}\left(L_{0}\right)$. For any $L_{0}<L_{0}^{*}$, there exists some $\delta \in \Delta_{L_{0}}$ such that $h(\delta)<0$ and, thus, $E g_{1}\left(\delta, \gamma^{*}\right)=-e\left(1-\lambda_{1} L_{0}\right)$ from (5.11). Since $e<a$, we have $-a\left(1+\nu_{1} L_{1}\right)<-e\left(1-\lambda_{1} L_{0}\right)$ for all $L_{1}$ and $L_{0}$. This implies

$$
\max _{\delta \in \Delta_{L_{0}}} E g_{1}\left(\delta, \gamma^{*}\right)=-e\left(1-\lambda_{1} L_{0}\right) \text { for every } L_{0}<L_{0}^{*}
$$

Similarly, for $L_{0}=L_{0}^{*}$

$$
\max _{\delta \in \Delta_{L_{0}^{*}}^{*}} E g_{1}\left(\delta, \gamma^{*}\right)=-e\left(1-\lambda_{1} L_{0}^{*}\right)
$$


and the solution $\delta^{*}$ of this maximization satisfies $L_{1}\left(\delta^{*}\right)=L_{1}\left(L_{0}^{*}\right)$. We have solved the subproblem $\max E g_{1}\left(\delta, \gamma^{*}\right)$ for every $L_{0} \geq 1$. From the arguments above, the test procedure $\delta^{*}$ of the inspector has the average run length $L_{0}\left(\delta^{*}\right)$ under $H_{0}$ which is a solution of

$$
\max _{L_{0} \geq 1} \begin{cases}-a\left(1+\nu_{1} L_{1}\left(L_{0}\right)\right) & \text { if } L_{0}>L_{0}^{*} \\ -e\left(1-\lambda_{1} L_{0}\right) & \text { if } L_{0}^{*} \geq L_{0} .\end{cases}
$$

It is easily seen that $L_{0}\left(\delta^{*}\right)=L_{0}^{*}$ and $L_{1}\left(\delta^{*}\right)=L_{1}\left(L_{0}^{*}\right)$ which completes the theorein (see Figure $4 b$ ).

This proposition shows again, like Theorem 4.4, that the inspectee will behave legally if the inspector plays his equilibrium strategy.

Eq. (5.7) corresponds to Eq. (4.6). Whereas, however, it can be seen easily that Eq. (4.6) has a unique solution under very general assumptions, this is not the case here: If one assumes reasonably $1 \leq L_{1}\left(L_{0}\right)<L_{0}$, then one sees immediately

$$
-b\left(1-\nu_{2} L_{1}\right)+f\left(1-\lambda_{2} L_{0}\right)<0 \text { for } b-f>\left(b \nu_{2}-f \lambda_{2}\right) \cdot L_{0}
$$

or, with $b \nu_{2}-f \lambda_{2}>0$,

$$
-b\left(1-\nu_{2} \mathrm{E}_{1}\right)+f\left(1-\lambda_{2} L_{0}\right)<0 \text { for } L_{0}<(b-f) /\left(b \nu_{2}-f \lambda_{2}\right)
$$

in this case the inspectee will always behave legally.

\section{Conclusions}

Let us consider once more the result of the fourth section where we assumed that both players' payoffs are independent of the stage where the game terminates. Theorem 4.3 shows that it suffices to optimize the probability of no detection for a given value of the false alarm probability $\alpha$ which is determined with the help of Eq. (4.6). This is important for several reasons: First, it is in line with standard statistical practice to proceed this way, and it permits the determination of optimal test procedures with the help of the Neyman-Pearson Lemma, see, e.g., [6].

One subtle point should be mentioned here: The application of the Neyman-Pearson Lemma requires a fixed alternative hypothesis which leads to the optimization problem

$$
\beta(\alpha)=\max _{\mu} \min _{\delta \in \Delta_{\alpha}} \beta(\delta, \mu)
$$

instead of (4.5). If, however, there exists a saddlepoint, then we can obtain an equilibrium point with the help of (6.1). This way, a series of practical problems have been solved, see, e.g., [1].

Second, for a given value of $\alpha$, the payoff parameters need not be known for the determination of the optimal test procedure. In fact, in many practical cases it is impossible to estimate even ranges of these parameters. Only if one wants to determine the optimal value of $\alpha$, then one needs to know the two ratios $b / f$ and $d / f$ of the inspectee's payoff parameters.

A similar picture is given by the result of the fifth section where the time aspect is important. Theorem 5.5 shows that it suffices to optimize the average run length under the alternative hypothesis for a given value of the average run length under the null hypothesis which is determined with the help of eq. (5.7). This result justifies again standard statistical practice, see, e.g., [9], even though there does not exist an equivalent of the Neyman-Pearson Lemma for sequential test procedures. 


\section{Acknowledgements}

We are grateful to anonymous referees for their very helpful comments. Akira Okada's work was supported in part by Tokyo Center for Economic Research (TCER) under the 1989-1990 Research Project Grant.

\section{References}

[1] R. Avenhaus, Safeguards Systems Analysis. Plenum Publisher, New York and London 1986

[2] R. Avenhaus, A. Okada, Inspector-Leadership Games with Incomplete Information. Discussion Paper No. 17, Zentrum für Interdisziplinäre Forschung an der Universität Bielefeld, July 1988

[3] Y.S. Chow, H. Robbins, and D. Siegmund, Great Expectations: The Theory of Optimal Stopping, Houghton Mifflin, Boston 1971

[4] M. Dresher, A Sampling Inspection Problem in Arms Control Agreements: A Game Theoretic Analysis. Memorandum No. RM-2972-ARPA, The RAND Corporation, Santa Monica, California 1962

[5] E. Höpfinger, Reliable Inspection Strategies. Vol. 17 of the Mathematical Systems in Economic Series, Anton Hain, Meisenheim am Glan (FRG) 1975; Zeitschr. Wahrsch. Theorie und verwandte Gebiete, Vol. 31, pp. 35-46 1974

[6] E.L. Lehmann, Testing Statistical Hypotheses. Wiley, New York 1959

[7] M. Maschler, A Price Leadership Method for Solving the Inspector's Non-ConstantSum Game. Nav. Res. Logistics Q. 13, pp, 11-33 1966

[8] M. Maschler, The Inspector's Non-Constant-Sum Game: Its Dependence on a System of Detectors. Nav. Res. Logistics Q. 14, pp. 275-290 1967

[9] G.B. Wetherill, Sampling Inspection and Quality Control. Chapman and Hall, London and New York, 2nd Edition 1982

\section{Annex: A Simple Sequential Test Problem}

Given the sequence $X_{1}, X_{2}, \ldots$ of independently, identically and normally distributed random variables, and the two hypotheses

$$
\begin{aligned}
& H_{0}: X_{i} \sim n\left(0, \sigma^{2}\right) \\
& H_{1}: X_{i} \sim n\left(\mu, \sigma^{2}\right), \mu>0, i=1,2, \ldots
\end{aligned}
$$

Let the sequential test procedure be defined as follows:

$$
\begin{aligned}
& \hat{X}_{i} \leq s: \text { continue } \\
& \hat{X}_{i}>s: \text { stop }, i=1,2, \ldots
\end{aligned}
$$

where $\hat{X}_{i}$ is an observation of the random variable $X_{i}, i=1,2, \ldots$. Let the probabilities $\alpha$ and $\beta$ of the errors first and second kind be given by

$$
\begin{gathered}
1-\alpha:=\operatorname{prob}\left(X_{i} \leq s \mid H_{0}\right)=\Phi\left(\frac{s}{\sigma}\right) \\
\beta:=\operatorname{prob}\left(X_{i} \leq s \mid H_{1}\right)=\Phi\left(\frac{s}{\sigma}-\frac{\mu}{\sigma}\right)=\Phi\left(U(1-\alpha)-\frac{\mu}{\sigma}\right)
\end{gathered}
$$


where $\Phi($.$) is the normal distribution function and U($.$) its inverse.$

The Average Run Length $L$ of the test is defined as

$$
L:=\sum_{i=1}^{\infty} i \cdot \operatorname{prob}\left(X_{i}>s\right) \cdot \prod_{j=1}^{i-1} \operatorname{prob}\left(X_{j} \leq s\right) .
$$

For $H_{0}$ we get

$$
L_{0}=\sum_{i=1}^{\infty} i \cdot \alpha \cdot(1-\alpha)^{i-1}=\frac{1}{\alpha}
$$

and for $H_{1}$

$$
L_{1}=\sum_{i=1}^{\infty} i \cdot(1-\beta) \cdot \beta^{i-1}=\frac{1}{1-\beta} .
$$

Therefore, with $U(1-\alpha)=-U(\alpha)$ we get the following relation between $L_{0}$ and $L_{1}$ :

$$
\frac{1}{L_{1}}=1-\beta=\Phi\left(\frac{\mu}{\sigma}+U(\alpha)\right)=\Phi\left(\frac{\mu}{\sigma}+U\left(\frac{1}{L_{0}}\right)\right)
$$

or, equivalently,

$$
U\left(\frac{1}{L_{1}}\right)=\frac{\mu}{\sigma}+U\left(\frac{1}{L_{0}}\right) .
$$

This relation between $L_{0}$ and $L_{1}$ has the following properties if we remember $\mu / \sigma>0$ and $U($.$) being strictly monotonically increasing with U(0)=-\infty, U(1)=\infty$ :

i) $L_{0}=1$ implies $L_{1}=1$

ii) $L_{1}$ increases monotonically with $L_{0}$

iii) $L_{1}<L_{0}$ for $L_{0}>1$.

These properties are typical for sequential tests, even though they cannot be shown as easily for tests other than those discussed here.

Akira OKADA:

Kyoto Institute of Economic Research

Kyoto University

Sakyo, Kyoto 606-01, Japan 